\title{
Tafamidis: a new treatment for ATTR cardiomyopathy
}

Transthyretin amyloid cardiomyopathy (ATTR-CM) is an underdiagnosed, life-threatening disease with no effective therapy. According to the results of the phase III ATTR-ACT trial presented at the 2018 ESC Congress, tafamidis - a drug used for the treatment of ATTR polyneuropathy (ATTR-PN) reduces mortality in patients with ATTR-CM.

ATTR-CM and ATTR-PN are caused by the aggregation of misfolded monomers of transthyretin (TTR) into fibrils in the heart and the peripheral nervous system, respectively. Cardiac amyloidosis leads to symptoms of heart failure (HF) that are often fatal.

Tafamidis, which was designed to bind to TTR and prevent its dissociation into monomers, was shown to slow neurological impairment in patients with ATTR-PN.
"Since the basic mechanisms underlying the development of ATTR-CM and ATTR-PN are similar, we hypothesized that tafamidis would be effective for affected cardiac patients," explains study investigator Mathew Maurer.

In the multicentre, international, double-blind trial, 441 patients with ATTR-CM were randomly assigned to receive tafamidis ( $20 \mathrm{mg}$ or $80 \mathrm{mg}$ ) or placebo for 30 months. Compared with placebo, tafamidis (doses combined) reduced allcause mortality by $30 \%$ (HR $0.70,95 \%$ CI $0.51-0.96)$ and the rate of cardiovascular-related hospitalizations by $32 \%$ (RR 0.68 , 95\% CI 0.56-0.81). At month 30, tafamidis was also associated with a lower decline in functional capacity — assessed by the 6-min walk test and a better health status compared with placebo.

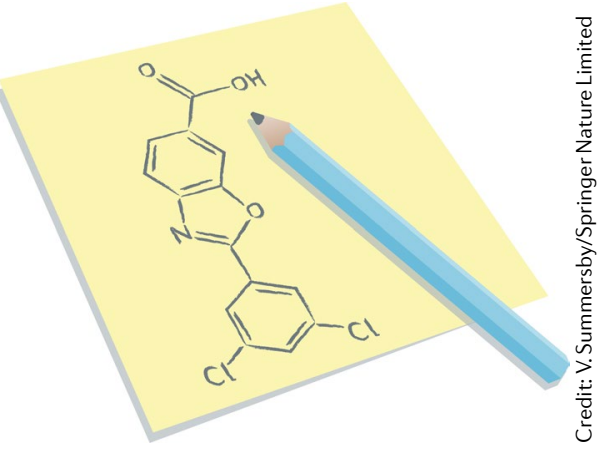

“The ATTR-ACT trial strongly supports the concept of phenotyping patients more carefully to identify the precise cause of their HF and treat the disease with specific therapies," comments Maurer. The team is now investigating the use of nuclear scintigraphy to screen patients with HF for TTR amyloidosis, in an effort to achieve a better and earlier diagnosis.

Alexandra Le Bras

patients more

carefully to identify the precise cause of their HF ORIGINAL ARTICLE Maurer, M. S. et al. Tafamidis treatment for patients with transthyretin amyloid cardiomyopathy. N. Engl. J. Med. https://doi.org/ 10.1056/NEJMoa1805689 (2018) FURTHER READING Gertz, M. A. et al. Pathophysiology and treatment of cardiac amyloidosis. Nat. Rev. Cardiol. 12, 91-102 (2015)

\section{Drug-coated balloons - another option for small-vessel disease}

Stent-free treatment with a drugcoated balloon (DCB) is noninferior to drug-eluting stents (DES) for de novo lesions in small coronary arteries, according to results from the BASKETSMALL 2 trial presented at the 2018 ESC Congress. Second-generation DES are the standard treatment for percutaneous intervention in coronary artery disease, but use of DES in small coronary arteries is associated with a higher risk of restenosis and lesion failure than in larger vessels. "Given the known high rates of adverse events with DES in small-vessel coronary artery disease, the option of DCB treatment as an alternative to DES is very welcome," remarks lead investigator Raban Jeger.

At present, DCBs are an established therapeutic option for in-stent restenosis, but data from randomized, controlled trials on DCBs in small coronary arteries are limited. Therefore, Jeger and colleagues initiated an open-label, randomized, noninferiority trial to assess the safety and efficacy of DCBs for the treatment of small-vessel coronary artery disease. In total, 758 patients with de novo lesions in small coronary arteries $(<3 \mathrm{~mm}$ in diameter) were randomly assigned to receive the SeQuent Please DCB (B. Braun Melsungen) or secondgeneration DES (everolimus-eluting Xience stent (Abbott Vascular) or paclitaxel-eluting Taxus Element stent (Boston Scientific)) after successful predilatation of the lesion with a standard balloon. All patients received dual antiplatelet therapy according to current guidelines.

The rate of major adverse cardiac events (MACE; cardiac-related death, nonfatal myocardial infarction, and target-vessel revascularization) after 1 year was similar in the DCB and DES groups (7.5\% versus $7.3 \%$; HR 0.97, 95\% Cl 0.58-1.64). Results

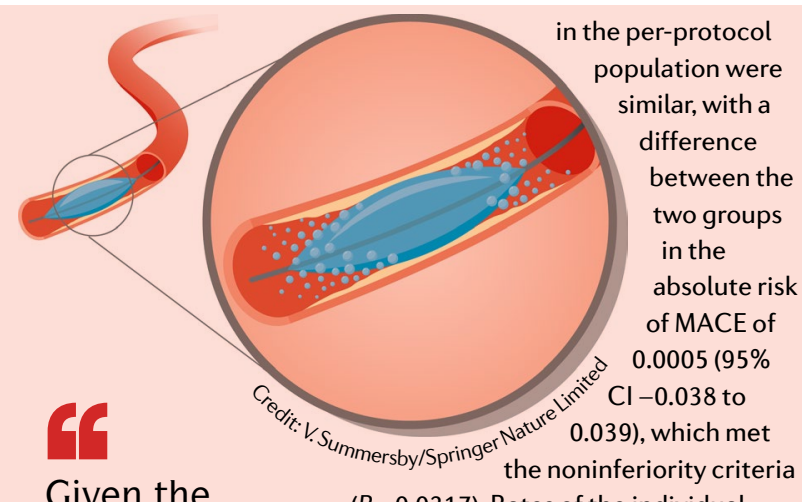

Given the known high rates of adverse events with DES in small-vessel coronary artery disease, the option of DCB treatment $\ldots$ is very welcome 5 $(P=0.0217)$. Rates of the individual components of the primary end point did not differ between the groups, and adverse events rates were low and similar in both groups.

Larger studies with a longer follow-up are needed to confirm these findings. Looking to the future, Jeger comments that the investigators will try to expand the DCB technology to other patient populations, such as patients with lesions in larger coronary arteries.

Irene Fernández-Ruiz

ORIGINAL ARTICLE Jeger, R. V. et al. Drug-coated balloons for small coronary artery disease (BASKET-SMALL 2): an open-label randomised non-inferiority trial. Lancet 392, 849-856 (2018) 